\title{
THE EXTENDED INVARIANCE PRINCIPLE APPLIED TO JOINT TIME-DELAY, FREQUENCY, AND DOA ESTIMATION
}

\author{
Felix Antreich ${ }^{*}$ Josef A. Nossek ${ }^{\star}$, Gonzalo Seco ${ }^{\dagger}$, and A. Lee Swindlehurst ${ }^{\ddagger}$ \\ German Aerospace Center (DLR), Inst. for Communications and Navigation, 82234 Wessling, Germany * \\ Inst. for Circuit Theory \& Signal Processing, Munich University of Technology (TUM), Germany \\ Dept. of Telecommunications \& Systems Engineering, Universitat Autnoma de Barcelona (UAB), Spain ${ }^{\dagger}$ \\ Dept. of Electrical Engineering \& Computer Science, The University of California, Irvine CA, U.S.A. ${ }^{\ddagger}$ \\ e-mail: felix.antreich@dlr.de*,nossek@nws.ei.tum.de ${ }^{\star}$, gonzalo.seco@uab.es ${ }^{\dagger}$, and swindle@uci.edu ${ }^{\ddagger}$
}

\begin{abstract}
This paper deals with the joint estimation of temporal (timedelay, Doppler frequency) and spatial (direction-of-arrival, DOA) parameters of several replicas of a known signal in an unknown spatially correlated field. Unstructured and structured models have been proposed in the literature. The former suffers from a severe performance degradation in some scenarios, whereas the latter involves huge complexity. It is shown how the extended invariance principle (EXIP) can be applied to obtain estimates with the quality of those of the structured model, but with the complexity of the unstructured one. We present a method to improve the quality of the timedelay and Doppler estimates obtained with an unstructured spatial model when an estimate of the DOAs is available. Exemplarily, simulation results for time-delay estimation for GPS (Global Positioning System) are included and confirm that our proposal approaches the Cramer-Rao lower bound (CRLB) of the structured model even when suboptimal DOA estimates obtained by ESPRIT are introduced.
\end{abstract}

Index Terms - Array signal processing, parameter estimation, direction of arrival estimation, Global Positioning System

\section{INTRODUCTION}

Channel estimation is important in many applications as MIMO channel characterization, radar, synchronization, and Global Navigation Satellite Systems (GNSS) like GPS (Global Positioning System). A simple unstructured data model has been used for such problems in order to have low complexity [1] and a solution for an unknown spatial field was given in [2]. On the other hand the structured data model provides better results [3,4] but has a high complexity and only seems to be easily manageable in the single path case [5]. The latter work already shows how the extended invariance principle (EXIP) can be applied to refine estimates for an unstructured model to achieve the performance available using a structured model.
This work extends the approach given in [5] to a multipath case. Applying EXIP, we derive an estimator that only depends on the directions-of-arrival (DOAs) of the signals. We attain a closed form solution that allows to correct the delay and Doppler estimates of the unstructured model once DOA estimates are available. In this work we will apply Unitary ESPRIT [6] for the DOA estimation. This two-step approach offers large flexibility since it is shown that an improvement of the delay and Doppler estimates can be achieved even when non-maximum likelihood (ML) estimates are introduced. This enables to use various rotational invariance techniques to obtain DOA estimates and still approach the Cramer-Rao lower bound (CRLB) of the timedelay and Doppler for the structured model.

In this work we apply this two-step approach for timedelay estimation of the line-of-sight signal (LOSS) for GPS. Here, precise time-delay estimation is needed for synchronization and thus accurate positioning, even under presence of severe multipath and interference.

\section{DATA MODEL}

We assume that $L$ narrowband planar wavefronts, $1 \leq \ell \leq L$ are impinging on an array of $M$ isotropic sensor elements. The noise-plus-interference corrupted baseband signal at the antenna output $\mathbf{y}(t) \in \mathbb{C}^{M \times 1}$ can be modelled as a superposition of $L$ wavefronts and additional temporally white Gaussian noise $\mathbf{n}(t) \in \mathbb{C}^{M \times 1}$, with zero-mean and unknown spatial covariance matrix $\mathbf{Q} \in \mathbb{C}^{M \times M}$.

\subsection{Structured Model}

For the structured data model we define the parameter vector $\boldsymbol{\theta}=\left[\operatorname{Re}\{\boldsymbol{\gamma}\}^{\mathrm{T}}, \operatorname{Im}\{\boldsymbol{\gamma}\}^{\mathrm{T}}, \boldsymbol{\phi}^{\mathrm{T}}, \boldsymbol{\vartheta}^{\mathrm{T}}, \boldsymbol{\tau}^{\mathrm{T}}, \boldsymbol{\nu}^{\mathrm{T}}\right]^{\mathrm{T}}$ on the domain $D_{\boldsymbol{\theta}}$ with the vector of complex amplitudes $\gamma=\left[\gamma_{1}, \ldots, \gamma_{\ell}, \ldots, \gamma_{L}\right]^{\mathrm{T}}$, the vector of azimuth angles $\phi=\left[\phi_{1}, \ldots, \phi_{\ell}, \ldots, \phi_{L}\right]^{\mathrm{T}}$, the vector of elevation angles $\boldsymbol{\vartheta}=\left[\vartheta_{1}, \ldots, \vartheta_{\ell}, \ldots, \vartheta_{L}\right]^{\mathrm{T}}$, the vector of time-delays $\boldsymbol{\tau}=$ 
$\left[\tau_{1}, \ldots, \tau_{\ell}, \ldots, \tau_{L}\right]^{\mathrm{T}}$, and the vector of Doppler frequencies $\boldsymbol{\nu}=\left[\nu_{1}, \ldots, \nu_{\ell}, \ldots, \nu_{L}\right]^{\mathrm{T}}$. Thus we can write

$$
\mathbf{y}(t)=\sum_{\ell=1}^{L} \mathbf{s}_{\ell}(t)+\mathbf{n}(t)
$$

where $\mathbf{s}_{\ell}(t)$ is given by

$$
\mathbf{s}_{\ell}(t)=\mathbf{a}_{\ell}\left(\phi_{\ell}, \vartheta_{\ell}\right) \gamma_{\ell} \mathrm{e}^{\mathrm{j} 2 \pi \nu_{\ell} t} c\left(t-\tau_{\ell}\right) .
$$

Here, $\mathbf{a}_{\ell}\left(\phi_{\ell}, \vartheta_{\ell}\right)$ denotes the steering vector of an antenna array and $c\left(t-\tau_{\ell}\right)$ denotes the pseudo-random-noise (PN) sequence with delay $\tau_{\ell}$.

\subsection{Unstructured Model}

For the simpler unstructured model we define the parameter vector $\boldsymbol{\xi}=\left[\operatorname{Re}\{\operatorname{vec}\{\mathbf{H}\}\}^{\mathrm{T}}, \operatorname{Im}\{\operatorname{vec}\{\mathbf{H}\}\}^{\mathrm{T}}, \boldsymbol{\tau}^{\mathrm{T}}, \boldsymbol{\nu}^{\mathrm{T}}\right]^{\mathrm{T}}$ on the domain $D_{\boldsymbol{\xi}}$ with the matrix of spatial signatures $\mathbf{H}=$ $\left[\begin{array}{lllll}\mathbf{h}_{1} & \cdots & \mathbf{h}_{\ell} & \cdots & \mathbf{h}_{L}\end{array}\right] \in \mathbb{C}^{M \times L}$. Here, $\operatorname{vec}\{\cdot\}$ denotes the vec operator, which vectorizes a matrix by stacking its columns. Thus we can alternatively write

$$
\mathbf{s}_{\ell}(t)=\mathbf{h}_{\ell} \mathrm{e}^{\mathrm{j} 2 \pi \nu_{\ell} t} c\left(t-\tau_{\ell}\right) .
$$

\section{MAXIMUM LIKELIHOOD (ML) ESTIMATION}

The spatial observations are collected at $N$ time instances, as $\mathbf{y}[n]=\mathbf{y}\left(n \cdot T_{s}\right)$ with $n=1,2, \ldots, N$. The channel parameters are assumed constant during the observation interval. Collecting the samples of the observation interval leads to

$$
\begin{aligned}
\mathbf{Y} & =[\mathbf{y}[1], \mathbf{y}[2], \ldots, \mathbf{y}[N]] \in \mathbb{C}^{M \times N}, \\
\mathbf{N} & =[\mathbf{n}[1], \mathbf{n}[2], \ldots, \mathbf{n}[N]] \in \mathbb{C}^{M \times N}, \\
\mathbf{S}(\boldsymbol{\xi}) & =[\mathbf{s}[1], \mathbf{s}[2], \ldots, \mathbf{s}[N]] \in \mathbb{C}^{M \times N} .
\end{aligned}
$$

Thus, the signal for the unstructured model can be written in matrix notation

$$
\mathbf{Y}=\mathbf{S}(\boldsymbol{\xi})+\mathbf{N}=\sum_{\ell=1}^{L} \mathbf{S}_{\ell}\left(\boldsymbol{\xi}_{\ell}\right)+\mathbf{N}=\mathbf{H}(\mathbf{C} \odot \mathbf{D})+\mathbf{N}
$$

where $\odot$ denotes the Hadamard-Schur product. Here, $\boldsymbol{\xi}_{\ell}=$ $\left[\operatorname{Re}\left\{\mathbf{h}_{\ell}\right\}^{\mathrm{T}}, \operatorname{Im}\left\{\mathbf{h}_{\ell}\right\}^{\mathrm{T}}, \tau_{\ell}, \nu_{\ell}\right]^{\mathrm{T}}$ contains the parameters of one wave, $\mathbf{C}=\left[\mathbf{c}\left(\tau_{1}\right) \cdots \mathbf{c}\left(\tau_{\ell}\right) \cdots \mathbf{c}\left(\tau_{L}\right)\right]^{\mathrm{T}} \in \mathbb{R}^{L \times N}$ contains the sampled $\mathrm{PN}$ sequence for each impinging wavefront $\mathbf{c}\left(\tau_{\ell}\right)$, and $\mathbf{D}=\left[\mathbf{d}\left(\nu_{1}\right) \cdots \mathbf{d}\left(\nu_{\ell}\right) \cdots \mathbf{d}\left(\nu_{L}\right)\right]^{\mathrm{T}} \in \mathbb{C}^{L \times N}$ contains the sampled Doppler frequencies for each impinging wavefront

$$
\mathbf{d}\left(\nu_{\ell}\right)=\left[\mathrm{e}^{\mathrm{j} 2 \pi \nu_{\ell} T_{s}}, \ldots, \mathrm{e}^{\mathrm{j} 2 \pi \nu_{\ell} n \cdot T_{s}}, \ldots, \mathrm{e}^{\mathrm{j} 2 \pi \nu_{\ell} N \cdot T_{s}}\right]^{\mathrm{T}} .
$$

The negative log-likelihood function for the unstructured data model within additive constants can be given [5]

$$
\Lambda(\boldsymbol{\xi}, \mathbf{Q})=N \cdot \log (\operatorname{det}(\mathbf{Q}))+\operatorname{tr}\left(\mathbf{Q}^{-1}(\mathbf{Y}-\mathbf{S}(\boldsymbol{\xi}))(\mathbf{Y}-\mathbf{S}(\boldsymbol{\xi}))^{\mathrm{H}}\right),
$$

where $(\cdot)^{\mathrm{H}}$ denotes complex conjugate transposition, $\operatorname{det}(\cdot)$ the determinant operation, and $\operatorname{tr}(\cdot)$ the trace operator. The ML estimate for $\mathbf{Q}$ is given by

$$
\hat{\mathbf{Q}}=\frac{1}{N}(\mathbf{Y}-\mathbf{S}(\boldsymbol{\xi}))(\mathbf{Y}-\mathbf{S}(\boldsymbol{\xi}))^{\mathrm{H}} .
$$

\section{SPACE-ALTERNATING GENERALIZED EXPECTATION MAXIMIZATION (SAGE) ALGORITHM}

In order to obtain the ML estimates $\hat{\xi}$ for the unstructured model we apply an iterative method, the SAGE algorithm [4]. We assume that $L$ is given. The expectation step (E-Step) can be derived

$$
\begin{aligned}
\hat{\mathbf{X}}_{\ell} & =\mathbf{Y}-\sum_{\substack{\ell^{\prime}=1 \\
\ell^{\prime} \neq \ell}}^{L} \mathbf{S}_{\ell^{\prime}}\left(\hat{\boldsymbol{\xi}}_{\ell^{\prime}}\right), \\
\hat{\mathbf{Q}} & =\frac{1}{N}(\mathbf{Y}-\mathbf{S}(\hat{\boldsymbol{\xi}}))(\mathbf{Y}-\mathbf{S}(\hat{\boldsymbol{\xi}}))^{\mathrm{H}},
\end{aligned}
$$

and for the maximization step (M-Step) we get

$$
\begin{aligned}
& \hat{\tau}_{\ell}=\arg \max _{\tau_{\ell}}\left\{\left\|\hat{\mathbf{Q}}^{-\frac{1}{2}} \hat{\mathbf{X}}_{\ell}\left(\mathbf{c}\left(\tau_{\ell}\right) \odot \mathbf{d}\left(\hat{\nu}_{\ell}\right)\right)^{*}\right\|_{2}^{2}\right\}, \\
& \hat{\nu}_{\ell}=\arg \max _{\nu_{\ell}}\left\{\left\|\hat{\mathbf{Q}}^{-\frac{1}{2}} \hat{\mathbf{X}}_{\ell}\left(\mathbf{c}\left(\hat{\tau}_{\ell}\right) \odot \mathbf{d}\left(\nu_{\ell}\right)\right)^{*}\right\|_{2}^{2}\right\}, \\
& \hat{\mathbf{h}}_{\ell}=\frac{\hat{\mathbf{X}}_{\ell}\left(\mathbf{c}\left(\hat{\tau}_{\ell}\right) \odot \mathbf{d}\left(\hat{\nu}_{\ell}\right)\right)^{*}}{N} .
\end{aligned}
$$

The parameters of each wavefront are estimated sequentially. The E-step and the M-step are performed iteratively for each wavefront until the algorithm converges. Instead of solving a $2 \cdot L$-dimensional non-linear optimization problem only 1 dimensional optimization procedures need to be solved. Initialization of the SAGE algorithm is achieved with successive interference cancellation starting with $\hat{\xi}=[0, \ldots, 0]^{\mathrm{T}}$ as described in [3]. For the covariance matrix $\mathbf{Q}$ the initial estimate is

$$
\hat{\mathbf{Q}}=\frac{1}{N} \mathbf{Y} \mathbf{Y}^{\mathrm{H}} \text {. }
$$

\section{EXTENDED INVARIANCE PRINCIPLE (EXIP)}

As a second step we invoke the EXIP $[7,5]$ in order to refine the ML estimates, $\hat{\xi}$, to achieve the performance using an ML estimate for the structured model, $\hat{\boldsymbol{\theta}}$, for which we would need to solve a $4 \cdot L$-dimensional non-linear problem, if solved directly.

Assuming that there exists a function $f$ which is one to one, satisfying

$$
\boldsymbol{\xi}=f(\boldsymbol{\theta}) \in D_{\boldsymbol{\xi}} \quad, \forall_{\boldsymbol{\theta}} \in D_{\boldsymbol{\theta}}
$$

and

$$
\lim _{N \rightarrow \infty} \hat{\boldsymbol{\xi}}=\lim _{N \rightarrow \infty} f(\hat{\boldsymbol{\theta}}) .
$$


Then

$$
\hat{\hat{\boldsymbol{\theta}}}=\arg \min _{\boldsymbol{\theta}}[\hat{\boldsymbol{\xi}}-f(\boldsymbol{\theta})]^{\mathrm{T}} \mathbf{W}[\hat{\boldsymbol{\xi}}-f(\boldsymbol{\theta})],
$$

is asymptotically (for large $N$ ) equivalent to the structured ML estimate $\hat{\boldsymbol{\theta}}$, where

$$
\mathbf{W}=\left.\mathrm{E}\left\{\frac{\partial \Lambda(\boldsymbol{\xi}, \mathbf{Q})}{\partial \boldsymbol{\xi} \partial \boldsymbol{\xi}^{T}}\right\}\right|_{\substack{\boldsymbol{\xi}=\hat{\boldsymbol{\xi}} \\ \mathbf{Q}=\mathbf{Q}}},
$$

and $\mathrm{E}\{\cdot\}$ denotes expectation.

Thus, for the problem at hand we can write

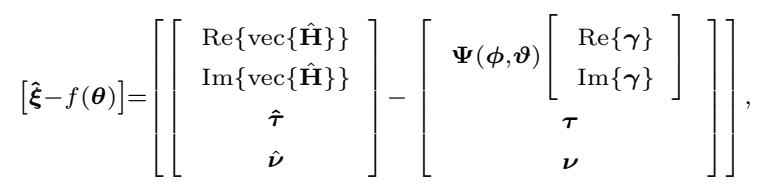

where

$$
\boldsymbol{\Psi}(\boldsymbol{\phi}, \boldsymbol{\vartheta})=\left[\begin{array}{cc}
\operatorname{Re}\left\{\mathbf{I}_{L} \square \mathbf{A}\right\} & -\operatorname{Im}\left\{\mathbf{I}_{L} \square \mathbf{A}\right\} \\
\operatorname{Im}\left\{\mathbf{I}_{L} \square \mathbf{A}\right\} & \operatorname{Re}\left\{\mathbf{I}_{L} \square \mathbf{A}\right\}
\end{array}\right] .
$$

Here, $\square$ denotes the Khatri-Rao product and $\mathbf{A}=$ $\left[\mathbf{a}_{1}\left(\phi_{1}, \vartheta_{1}\right) \cdots \mathbf{a}_{\ell}\left(\phi_{\ell}, \vartheta_{\ell}\right) \cdots \mathbf{a}_{L}\left(\phi_{L}, \vartheta_{L}\right)\right] \in \mathbb{C}^{M \times L}$. Further, we define

$$
\mathbf{W}=\left[\begin{array}{ccc}
\operatorname{Re}\left\{\mathbf{W}_{1}\right\} & -\operatorname{Im}\left\{\mathbf{W}_{1}\right\} & \operatorname{Re}\left\{\mathbf{W}_{3}\right\} \\
\operatorname{Im}\left\{\mathbf{W}_{1}\right\} & \operatorname{Re}\left\{\mathbf{W}_{1}\right\} & \operatorname{Im}\left\{\mathbf{W}_{3}\right\} \\
\operatorname{Re}\left\{\mathbf{W}_{3}\right\}^{\mathrm{T}} & \operatorname{Im}\left\{\mathbf{W}_{3}\right\}^{\mathrm{T}} & \operatorname{Re}\left\{\mathbf{W}_{2}\right\}
\end{array}\right],
$$

with

$$
\begin{gathered}
\mathbf{W}_{1}=2 \cdot\left((\mathbf{C} \odot \mathbf{D})(\mathbf{C} \odot \mathbf{D})^{\mathrm{H}}\right) \otimes \hat{\mathbf{Q}}^{-1}, \\
\mathbf{W}_{2}=2 \cdot\left(\mathbf{\Upsilon}^{\mathrm{H}} \mathbf{\Upsilon}\right) \odot\left([\mathbf{e} \mathbf{e}] \otimes\left(\hat{\mathbf{H}}^{\mathrm{H}} \hat{\mathbf{Q}}^{-1} \hat{\mathbf{H}}\right)\right), \\
\mathbf{W}_{3}=\left[\begin{array}{c}
2 \cdot\left(\mathbf{e}^{\mathrm{T}} \otimes\left(\hat{\mathbf{Q}}^{-1} \hat{\mathbf{H}}\right)\right) \operatorname{diag}\left\{\left(\mathbf{c}\left(\hat{\tau}_{1}\right) \odot \mathbf{d}\left(\hat{\nu}_{1}\right)\right)^{\mathrm{H}} \boldsymbol{\Upsilon}\right\} \\
\vdots \\
2 \cdot\left(\mathbf{e}^{\mathrm{T}} \otimes\left(\hat{\mathbf{Q}}^{-1} \hat{\mathbf{H}}\right)\right) \operatorname{diag}\left\{\left(\mathbf{c}\left(\hat{\tau}_{\ell}\right) \odot \mathbf{d}\left(\hat{\nu}_{\ell}\right)\right)^{\mathrm{H}} \boldsymbol{\Upsilon}\right\} \\
\vdots \\
2 \cdot\left(\mathbf{e}^{\mathrm{T}} \otimes\left(\hat{\mathbf{Q}}^{-1} \hat{\mathbf{H}}\right)\right) \operatorname{diag}\left\{\left(\mathbf{c}\left(\hat{\tau}_{L}\right) \odot \mathbf{d}\left(\hat{\nu}_{L}\right)\right)^{\mathrm{H}} \mathbf{\Upsilon}\right\}
\end{array}\right] .
\end{gathered}
$$

Here, $\otimes$ denotes the Kronecker product, $\operatorname{diag}\{\cdot\}$ defines a diagonal matrix, $\mathbf{e}=\left[\begin{array}{ll}1 & 1\end{array}\right]^{\mathrm{T}}$, and

$$
\begin{aligned}
& \mathbf{\Upsilon}=\left[\frac{\partial\left(\mathbf{c}\left(\hat{\tau}_{1}\right) \odot \mathbf{d}\left(\hat{\nu}_{1}\right)\right)}{\partial \hat{\tau}_{1}} \ldots \frac{\partial\left(\mathbf{c}\left(\hat{\tau}_{\ell}\right) \odot \mathbf{d}\left(\hat{\nu}_{\ell}\right)\right)}{\partial \hat{\tau}_{\ell}} \ldots \frac{\partial\left(\mathbf{c}\left(\hat{\tau}_{L}\right) \odot \mathbf{d}\left(\hat{\nu}_{L}\right)\right)}{\partial \hat{\tau}_{L}}\right. \\
& \left.\frac{\partial\left(\mathbf{c}\left(\hat{\tau}_{1}\right) \odot \mathbf{d}\left(\hat{\nu}_{1}\right)\right)}{\partial \hat{\nu}_{1}} \ldots \frac{\partial\left(\mathbf{c}\left(\hat{\ell}_{\ell}\right) \odot \mathbf{d}\left(\hat{\nu}_{\ell}\right)\right)}{\partial \hat{\nu}_{\ell}} \ldots \frac{\partial\left(\mathbf{c}\left(\hat{\tau}_{L}\right) \odot \mathbf{d}\left(\hat{\nu}_{L}\right)\right)}{\partial \hat{\nu}_{L}}\right] .
\end{aligned}
$$

Thus, applying (21) to (19) and then minimizing (19) with respect to $\tau$ and $\nu$ leads to the refined estimates

$$
\begin{aligned}
{\left[\begin{array}{c}
\hat{\hat{\boldsymbol{\tau}}} \\
\hat{\hat{\boldsymbol{\nu}}}
\end{array}\right] } & =\left[\begin{array}{c}
\hat{\boldsymbol{\tau}} \\
\hat{\boldsymbol{\nu}}
\end{array}\right]+\operatorname{Re}\left\{\mathbf{W}_{2}\right\}^{-1}\left[\operatorname{Re}\left\{\mathbf{W}_{3}\right\}^{\mathrm{T}} \operatorname{Im}\left\{\mathbf{W}_{3}\right\}^{\mathrm{T}}\right] \\
& \cdot\left[\left[\begin{array}{c}
\operatorname{Re}\{\operatorname{vec}\{\hat{\mathbf{H}}\}\} \\
\operatorname{Im}\{\operatorname{vec}\{\hat{\mathbf{H}}\}\}
\end{array}\right]-\boldsymbol{\Psi}(\hat{\hat{\boldsymbol{\phi}}}, \hat{\hat{\boldsymbol{\vartheta}}})\left[\begin{array}{l}
\operatorname{Re}\{\hat{\hat{\gamma}}\} \\
\operatorname{Im}\{\hat{\hat{\gamma}}\}
\end{array}\right]\right]
\end{aligned}
$$

Substituting (28) in (21) and then applying to (19) leads to

$$
\begin{aligned}
{\left[\begin{array}{l}
\operatorname{Re}\{\hat{\hat{\gamma}}\} \\
\operatorname{Im}\{\hat{\hat{\gamma}}\}
\end{array}\right] } & =\left(\boldsymbol{\Psi}^{\mathrm{T}}(\hat{\hat{\boldsymbol{\phi}}}, \hat{\hat{\boldsymbol{\vartheta}}}) \boldsymbol{\Omega} \boldsymbol{\Psi}(\hat{\hat{\boldsymbol{\phi}}}, \hat{\hat{\boldsymbol{\vartheta}}})\right)^{-1} \\
& \cdot \boldsymbol{\Psi}^{\mathrm{T}}(\hat{\hat{\boldsymbol{\phi}}}, \hat{\hat{\boldsymbol{\vartheta}}}) \boldsymbol{\Omega}\left[\begin{array}{c}
\operatorname{Re}\{\operatorname{vec}\{\hat{\mathbf{H}}\}\} \\
\operatorname{Im}\{\operatorname{vec}\{\hat{\mathbf{H}}\}\}
\end{array}\right],
\end{aligned}
$$

where

$$
\begin{aligned}
& \boldsymbol{\Omega}=\left[\left[\begin{array}{cc}
\operatorname{Re}\left\{\mathbf{W}_{1}\right\} & -\operatorname{Im}\left\{\mathbf{W}_{1}\right\} \\
\operatorname{Im}\left\{\mathbf{W}_{1}\right\} & \operatorname{Re}\left\{\mathbf{W}_{1}\right\}
\end{array}\right]\right. \\
& \left.-\left[\begin{array}{c}
\operatorname{Re}\left\{\mathbf{W}_{3}\right\} \\
\operatorname{Im}\left\{\mathbf{W}_{3}\right\}
\end{array}\right] \operatorname{Re}\left\{\mathbf{W}_{2}\right\}^{-1}\left[\operatorname{Re}\left\{\mathbf{W}_{3}\right\}^{\mathrm{T}} \operatorname{Im}\left\{\mathbf{W}_{3}\right\}^{\mathrm{T}}\right]\right] .
\end{aligned}
$$

Finally, $\hat{\hat{\phi}}$ and $\hat{\hat{\vartheta}}$ can be obtained by

$$
\begin{aligned}
& (\hat{\hat{\phi}}, \hat{\hat{\vartheta}})=\arg \max _{\boldsymbol{\phi}, \boldsymbol{\vartheta}}\left\{\left[\begin{array}{c}
\operatorname{Re}\{\operatorname{vec}\{\hat{\mathbf{H}}\}\} \\
\operatorname{Im}\{\operatorname{vec}\{\hat{\mathbf{H}}\}\}
\end{array}\right]^{\mathrm{T}} \boldsymbol{\Omega} \boldsymbol{\Psi}(\boldsymbol{\phi}, \boldsymbol{\vartheta})\right. \\
& \left.\cdot\left(\boldsymbol{\Psi}^{\mathrm{T}}(\boldsymbol{\phi}, \boldsymbol{\vartheta}) \boldsymbol{\Omega} \boldsymbol{\Psi}(\boldsymbol{\phi}, \boldsymbol{\vartheta})\right)^{-1} \boldsymbol{\Psi}^{\mathrm{T}}(\boldsymbol{\phi}, \boldsymbol{\vartheta}) \boldsymbol{\Omega}\left[\begin{array}{c}
\operatorname{Re}\{\operatorname{vec}\{\hat{\mathbf{H}}\}\} \\
\operatorname{Im}\{\operatorname{vec}\{\hat{\mathbf{H}}\}\}
\end{array}\right]\right\} .
\end{aligned}
$$

Thus, (31) provides estimates of the DOAs that are asymptotically equivalent to the ML estimates of the structured model. The estimates $\hat{\hat{\phi}}$ and $\hat{\hat{\vartheta}}$ are introduced to (29) and (28) improving the delay and Doppler estimates. The maximization of (31) in general is complex unless particular array geometries are employed. Subspace fitting methods can be used, while maintaining the same asymptotic performance.

Instead of solving (31) we apply Unitary ESPRIT with structured least squares (SLS) [6] and spatial smoothing. We use the pre-whitened covariance estimate

$$
\hat{\mathbf{R}}=\hat{\mathbf{Q}}^{-\frac{1}{2}} \hat{\mathbf{H}}\left(\hat{\mathbf{Q}}^{-\frac{1}{2}} \hat{\mathbf{H}}\right)^{\mathrm{H}} .
$$

The important result, as we will see next, is that the DOA estimates obtained by ESPRIT (which in general underperform (31), but the analysis is out of the scope of this paper) can be introduced to (29) and (28), and thus lead to a significant improvement of the delay and Doppler estimates.

\section{SIMULATION RESULTS}

We assume a centro-symmetric [6] uniform rectangular array (URA) with $M=9$ isotropic sensor elements with halfwavelength spacing. The channel parameters are assumed constant during the observation interval $T_{N}$ and the one-sided bandwidth of the signal is $B=1.023 \mathrm{MHz}$. For the PN sequence we apply Gold codes [8] as used for the GPS C/A code with code period $T=1 \mathrm{~ms}, 1023$ chips per code period each with a time duration $T_{c}=977.52 \mathrm{~ns}$. Signal-to-noise ratio (SNR) denotes the LOSS-to-noise ratio. The effective SNR in $\mathrm{dB}$ can be obtained by

$$
\mathrm{SNR}=\mathrm{C} / \mathrm{N}_{0}-10 \cdot \log _{10}(2 \cdot B)+10 \cdot \log _{10}\left(N_{c}\right),
$$


whereas $\mathrm{C} / \mathrm{N}_{0}$ in $\mathrm{dB}-\mathrm{Hz}$ denotes the carrier-to-noise density ratio and $N_{c} \in \mathbb{N}$ is the number of code periods within $T_{N}=N_{c} T$. We assume C $/ \mathrm{N}_{0}=40.3 \mathrm{~dB}-\mathrm{Hz}$ and $N_{c}=20$ which leads to $\mathrm{SNR}=-9.8 \mathrm{~dB}$. We analyze the behavior of our approach for a single reflective multipath as a function of its relative delay to the LOSS $(L=2)$. In the following parameters with the subscript 1 refer to the LOSS and parameters with the subscript 2 refer to the reflection. The reflected multipath and the LOSS are considered to be in-phase and the signal-to-multipath ratio (SMR) is $5 \mathrm{~dB}$. Further, we assume a temporally white Gaussian interference with interferenceto-signal ratio (ISR) of $40 \mathrm{~dB}$ which is responsible for the spatial covariance of the noise plus interference field. The interference is uncorrelated with the signals and the noise. The DOAs for the LOSS and the multipath are $\phi_{1}=50^{\circ}$, $\phi_{2}=102^{\circ}, \vartheta_{1}=40^{\circ}, \vartheta_{2}=26^{\circ}$ and for the interference are $\phi_{I}=150^{\circ}, \vartheta_{I}=6^{\circ}$. We define $\Delta \tau=\left|\tau_{1}-\tau_{2}\right| / T_{c}$ and $\Delta \nu=\left|\nu_{1}-\nu_{2}\right| \cdot T_{N}$.

Fig. 1 and Fig. 2 depict the root mean square estimation error (RMSE) and CRLB of $\hat{\tau}_{1}$ and $\hat{\hat{\tau}}_{1}$ for $\Delta \nu=0$ and $\Delta \nu=0.5$ respectively. In Fig. 1 SAGE for small $\Delta \tau$ is biased and the RMSE $\hat{\tau}_{1}$ is below $\sqrt{\operatorname{CRLB}\left(\hat{\tau}_{1}\right)}$, since the interference cancellation in the E-step (11) is not able to separate the two waves if $\Delta \tau$ and $\Delta \nu$ are very small. In these cases the estimate of one hidden data space, $\hat{\mathbf{X}}_{1}$ is an estimate for a superposition of the two wavefronts and the other, $\hat{\mathbf{X}}_{2}$ includes only noise.

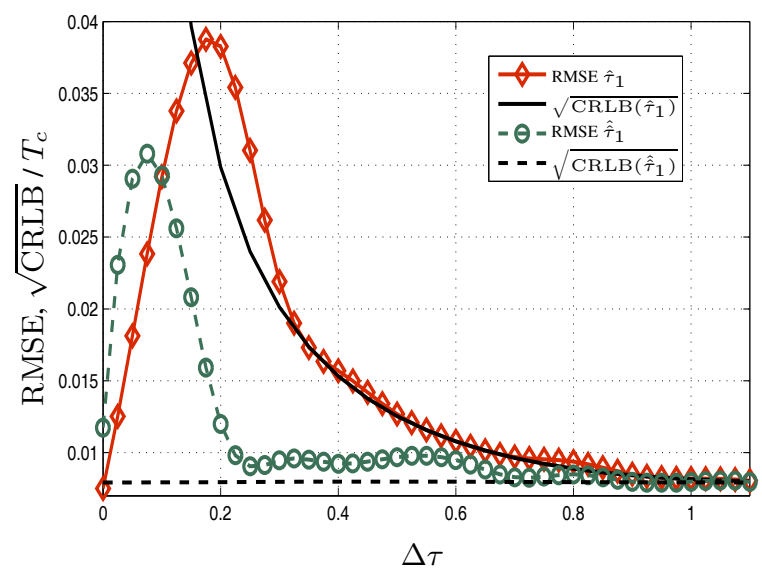

Fig. 1. RMSE of $\hat{\tau}_{1}$ and of $\hat{\hat{\tau}}_{1}$ versus $\Delta \tau$ for $\Delta \nu=0$

\section{CONCLUSIONS}

In this work we proposed a two-step approach to achieve estimates for a structured model for the multipath case in an unknown spatially colored field. We applied the EXIP to refine estimates achieved by an iterative ML estimator for an unstructured model, when an estimate of the DOAs is available.

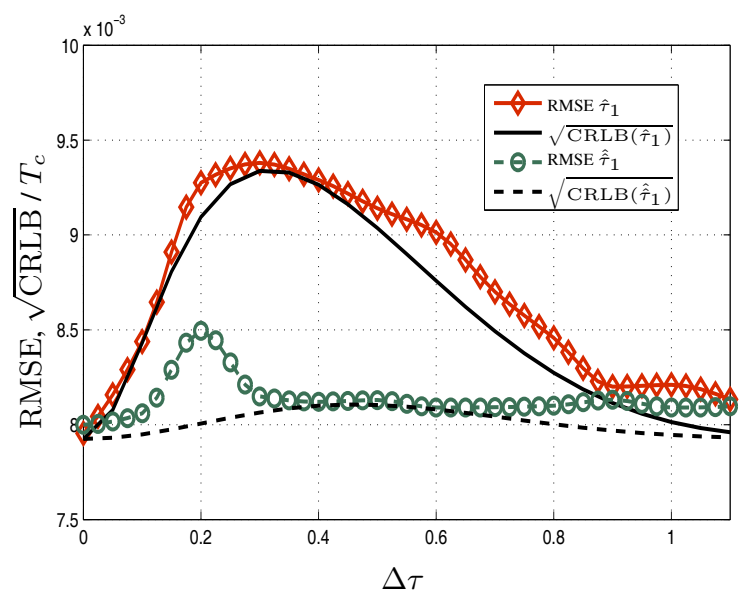

Fig. 2. RMSE of $\hat{\tau}_{1}$ and $\hat{\hat{\tau}}_{1}$ versus $\Delta \tau$ for $\Delta \nu=0.5$

Exemplarily, simulation results for time-delay estimation in GPS are shown which confirm that the proposed two-step approach attains the CRLB of the structured model even when suboptimal DOA estimates obtained by Unitary ESPRIT are introduced.

\section{REFERENCES}

[1] A. L. Swindlehurst, "Time Delay and Spatial Signature Estimation Using Known Asynchronous Signals," IEEE Transactions on Signal Processing, vol. 46, no. 2, February 1998.

[2] G. Seco-Granados, Antenna Arrays for Multipath and Interference Mitigation in GNSS Receivers, Ph.D. thesis, Department of Signal Signal Theory and Communications, Universitat Politècnica de Catalunya, 2000.

[3] F. Antreich, J. A. Nossek, and W. Utschick, "Maximum Likelihood Delay Estimation in a Navigation Receiver for Aeronautical Applications," Aerospace Science and Technology, doi:10.1016/j.ast.2007.06.005, 2007.

[4] B. H. Fleury, M. Tschudin, R. Heddergott, D. Dahlhaus, and K. I. Pedersen, "Channel Parameter Estimation in Mobile Radio Environments Using the SAGE Algorithm," IEEE Journal on Selected Areas in Communications, vol. 17, no. 3, March 1999.

[5] A. L. Swindlehurst and P. Stoica, "Maximum Likelihood Methods in Radar Array Signal Processing," Proceedings of the IEEE, vol. 86, no. 2, February 1998.

[6] M. Haardt, Efficient One-, Two-, and Multidimensional HighResolution Array Signal Processing, Ph.D. thesis, Department Electrical Engineering of Munich University of Technology, 1996.

[7] P. Stoica and T. Söderström, "On Reparametrization of Loss Functions Used in Estimation and the Invariance Principle," Signal Processing, vol. 17, pp. 383-387, August 1989.

[8] B. W. Parkinson and J. J. Spilker, Global Positioning System: Theory and Applications, vol. 1, Progress in Astronautics and Aeronautics, 1996. 